\title{
Nistagmo secundario a albinismo con compromiso ocular en paciente femenina
}

\author{
Nystagmus secondary to albinism with ocular involvement in a female
}

\author{
Luisa Schonhaut B. ${ }^{a}$, Joanna Britzmann L. ${ }^{b}$, Mario Zanolli S.c, \\ Jovanka Pavlov N. ${ }^{\mathrm{a}}$, Trinidad Hasbun Z. ${ }^{\mathrm{d}, \mathrm{e}}$, Gabriela Repetto L. ${ }^{\mathrm{a}}$
}

\begin{abstract}
aDepartamento de Pediatría, Facultad de Medicina, Clínica Alemana Universidad del Desarrollo. Santiago, Chile bServicio de Pediatría, Hospital Dr. Luis Calvo Mackenna. Santiago, Chile

'Departamento de Cirugía. Servicio de Oftalmología. Facultad de Medicina, Clínica Alemana Universidad del Desarrollo. Santiago, Chile ¿Departamento de Cirugía. Servicio de Dermatología. Facultad de Medicina, Clínica Alemana Universidad del Desarrollo. Santiago, Chile eServicio de Dermatología, Hospital Exequiel González Cortés. Santiago, Chile
\end{abstract}

Recibido: 10 de mayo de 2019; Aceptado: 6 de enero de 2020

\section{¿Qué se sabe del tema que trata este estudio?}

El nistagmo infantil es un desafío diagnóstico para el pediatra. El albinismo es una de sus principales causas, siendo difícil de sospechar en ausencia de compromiso cutáneo evidente, especialmente en pacientes femeninas.
¿Qué aporta este estudio a lo ya conocido?

A partir del caso de una lactante femenina que presenta nistagmo secundario a albinismo ocular aislado, se discute el enfoque diagnóstico clínico, enfatizando la relevancia de la pesquisa oportuna e intervención multidisciplinaria.

\section{Resumen}

El nistagmo infantil es infrecuente y representa un desafío diagnóstico para el pediatra. El albinismo es una de sus principales causas, siendo difícil de sospechar en ausencia de compromiso cutáneo evidente, especialmente en pacientes femeninas, debido a que tipo de herencia del albinismo ocular. Objetivo: Describir un caso de nistagmo secundario a albinismo con compromiso ocular aislado en paciente femenina, para discutir el enfoque diagnóstico pediátrico. Caso Clínico: Paciente femenino de 3 semanas de vida, sin antecedentes mórbidos, derivada a neuropediatra y oftalmólogo por movimientos oculares paroxísticos desde las 2 semanas, con estudio con electroencefalograma e imágenes cerebrales normales. A los 3 meses se confirmó translucencia iridiana, nistagmo y astigmatismo hipermetrópico. La valuación dermatológica descartó compromiso cutáneo. Evolucionó con inclinación cefálica hacia abajo y retraso del desarrollo de la coordinación, fue manejada con lentes
Palabras clave:

Albinismo;

albinismo ocular;

nistagmo;

nistagmo infantil;

tortícolis congénita

Correspondencia:

Mario Zanolli de S

mzanolli@alemana.cl 
de corrección y kinesioterapia. A los 3 años, destacaba mejoría de la agudeza visual, disminución del nistagmo y neurodesarrollo normal. La evaluación oftalmológica de ambos padres fue normal y no había antecedentes de nistagmo o albinismo en la familia. Por decisión de los padres no se realizó estudio genético. Conclusión: El diagnóstico de nistagmo secundario a compromiso ocular del albinismo, aún en ausencia de afección cutánea, es clínico; el estudio genético permite confirmar la etiología, sin ser un examen imprescindible, a menos que se considere la planificación familiar. La pesquisa oportuna e intervención multidisciplinaria determinan un mejor pronóstico.

\section{Keywords:}

Nystagmus; Ocular albinism; albinism; Infantile nystagmus; congenital torticollis tive: To describe a case of nystagmus secondary to albinism with isolated ocular involvement in a female patient, in order to provide tools for pediatric approach and diagnosis. Clinical Case: Threeweeks-old female patient, without morbid history, referred to a pediatric neurosurgeon and ophthalmologist due to paroxysmal eye movements since 2 weeks of age. The electroencephalogram and brain images were normal. In follow-up monitoring at 3 months, iris translucency, nystagmus, and hypermetropic astigmatism were confirmed. Dermatologic evaluation ruled out cutaneous involvement. The patient developed cephalic downward inclination and coordination development delay was confirmed, the patient was handled with corrective lenses and kinesiotherapy. In follow-up monitoring at 3 years, there was an improvement in visual acuity, decreased nystagmus and normal neurodevelopment. The ophthalmological evaluation of both parents was normal and there was no history of nystagmus or albinism in the family. Upon her parents' decision, no genetic study was carried out. Conclusion: The diagnosis of nystagmus secondary to ocular albinism, even in the absence of cutaneous involvement, is clinical. The genetic study allows confirming the etiology, without being an essential examination, unless family planning is considered. Timely research and multidisciplinary intervention determine a better prognosis.

\section{Introducción}

El nistagmo corresponde al movimiento ocular constante, rítmico, involuntario $y$, generalmente conjugado de ambos ojos, que puede ser en sentido horizontal, vertical u oblicuo. Se clasifica en congénito o infantil si está presente al nacimiento o se desarrolla durante los primeros meses de vida, y en adquirido, si aparece después de este periodo ${ }^{1}$. Se describe que el $87 \%$ de los nistagmos congénitos son diagnosticados antes de los 6 meses de edad ${ }^{2}$. Su relevancia clínica radica en el compromiso de la función visual, que dificulta el contacto visual y se asocia a problemas en el desarrollo psicomotor. Además, los individuos afectados suelen adoptar posturas cervicales anómalas o balanceos como medio para bloquear el movimiento ocular constante y consecuente disminución del nistagmus ${ }^{2-4}$.

El nistagmo es muy infrecuente; en Inglaterra se describe una prevalencia de 24 por 10.000 habitantes en población general, y de 16,6 por 10.000 menores de $18 \mathrm{años}^{5}$, mientras que, en Estado Unidos, la tasa reportada es de 35,3 por 10.000 en preescolares ${ }^{6}$.

El nistagmo infantil o congénito se asocia con una gran variedad de patologías oculares: defectos estructurales del globo ocular, opacidad de medios y alteraciones de la retina, y en menor frecuencia, a patologías a nivel del sistema nervioso central ${ }^{7,8}$. Se estima que alrededor del 10\% son idiopáticos o de causa desconocida $^{1}$. En niños, el albinismo es la causa individual más frecuente $e^{5,7,8}$.

El albinismo es un grupo heterogéneo de alteraciones pigmentarias, que afectan los procesos de biosíntesis del polímero de la melanina, pigmento producido por células ectodérmicas especializadas llamadas melanocitos ${ }^{9}$. Los melanocitos se diferencian, según el linaje ectodérmico, en cutáneos (fanéreos y piel) o extra cutáneos (oculares y cocleares). Todos los pacientes que padecen de albinismo en alguna de sus formas tienen compromiso oftalmológico, incluyendo por nistagmo, fotofobia, transiluminación del iris, menor agudeza visual e hipoplasia de la fóvea ${ }^{10}$. En el albinismo oculocutáneo (AOC) están involucrados además la piel y cabello, mientras que en el albinismo ocular (AO) el compromiso es sólo de los ojos ${ }^{9,11,12}$.

La prevalencia del albinismo varía ampliamente en los distintos grupos étnicos. En forma global el AOC afecta a 1 por 17.000 individuos de la población ge- 
neral, determinando que 1 por cada 70 personas son portadores de algún gen recesivo del $\mathrm{AOC}^{13}$. Estudios en niños en países nórdicos muestran prevalencias más altas, alcanzando 7 por 1.000 escolares $^{14}$. En el caso del AO la frecuencia estimada es de 1 en in 60.000 nacidos vivos $^{15}$. La diferencia en la prevalencia entre los dos tipos de albinismo se debe al tipo de herencia; mientras el AOC es de herencia autosómica recesiva, el AO generalmente es de herencia ligada al cromosoma $\mathrm{X}$, afectando predominantemente pacientes de sexo masculino $^{12}$.

Debido a su baja frecuencia y a su clínica heterogénea, el diagnóstico de nistagmo congénito a nivel de pediatras resulta complejo, especialmente en ausencia de hipopigmentación de la piel. El objetivo del presente manuscrito es describir un caso de nistagmo secundario a albinismo en una paciente de sexo femenino para discutir el enfoque diagnóstico pediátrico.

\section{Caso Clínico}

Lactante de sexo femenino, sin antecedentes perinatales ni familiares de relevancia. A partir de las 2 semanas de vida los padres notaron movimientos oculares paroxísticos en somnolencia, sueño y luego también en vigilia, por lo que fue evaluada por neuropediatra. Con el objeto de descartar síndrome convulsivo se solicitó electroencefalograma y ecografía cerebral que fueron normales. Debido a la evolución de movimientos oculares paroxísticos a un nistagmo horizontal de amplitud y frecuencia moderada, fue reevaluada por oftalmólogo, describiéndose transiluminación iridiana bilateral simétrica, hipoplasia macular, hipopigmentación retinal difusa y nervios ópticos grises, asociado a astigmatismo hipermetrópico (figura 1). Con estos hallazgos fue derivada a dermatología, descartándose hipopigmentación de piel o pelo. En cuanto a su desarrollo, destacaba tendencia a la inclinación cefálica hacia abajo, asociada a retraso de la coordinación.

La evaluación oftalmológica y autofluorescencia realizada en ambos padres descartó presencia de compromiso ocular y en la historia familiar no se encontraron otros casos de albinismo ni de nistagmo. En evaluación por genetista se sugirió estudio genético, el cual no se realizó por decisión de los padres.

La paciente se manejó con lentes ópticos de corrección y kinesioterapia para alineamiento cervical. En la última evaluación pediátrica y oftalmológica realizada a la edad de 3 años, detacaba una evolución oftalmológica favorable, con mejoría de la agudeza visual, disminución del nistagmo y adecuada progresión del desarrollo de la coordinación, motricidad fina y postura.

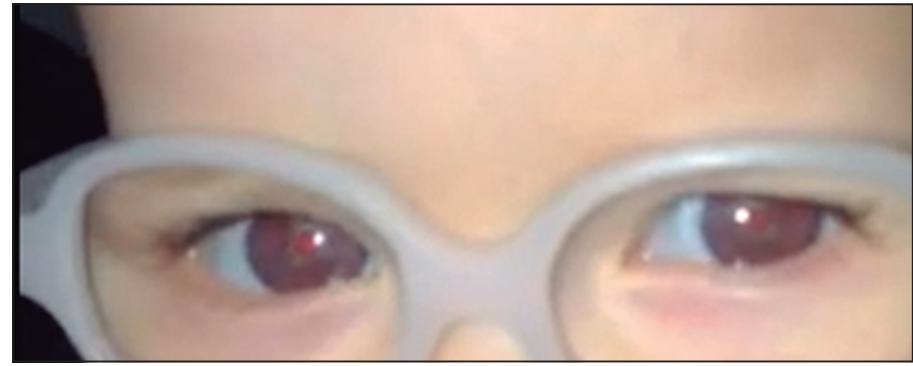

Figura 1. Evaluación visual: nótese translucencia iridiana.

\section{Discusión}

El nistagmo en lactantes y niños es una causa inhabitual de consulta. La orientación diagnóstica inicial es compleja, tal como el caso presentado, en que, en las primeras semanas de vida no era evidente que los movimientos oculares paroxísticos correspondieran a nistagmo. En nuestra paciente se descartó compromiso neurológico a través de estudio por imágenes y electroencefalograma. Una vez identificado el nistagmo, el objetivo fue definir si este era de origen ocular o sensorial, neurológico o idiopático (figura 2). Entre las causas de nistagmo, las más frecuentes son las oculares y entre estas predomina el albinismo $0^{5,7,8}$.

El diagnóstico de compromiso ocular en albinismo es clínico, fundamentado en la triada consistente en nistagmo, transiluminación del iris e hipoplasia de la fóvea ${ }^{10}$. En el examen oftalmológico se suele objetivar además una reducción de la agudeza visual, errores refractivos, alteraciones en iris, hipoplasia de la mácula, alteraciones en los nervios y en el quiasma óptico ${ }^{1}$. Frente a un examen ocular característico, no es necesario la complementación con exámenes adicionales. En el examen físico general, suele encentrarse a su vez hipopigmentación de piel y fanéreos, condición que no siempre está presente, ya que existen tipos de albinismo exclusivamente con manifestaciones oculares ${ }^{12}$.

$\mathrm{El} \mathrm{AO}$ es una condición rara, en la que no existe compromiso cutáneo. Debido a la herencia es ligada al cromosoma $\mathrm{X}$, esta condición predomina en pacientes masculinos ${ }^{12,16}$. Las mujeres tienen un cromosoma $\mathrm{X}$ inactivado al azar, siendo el "remanente sano" suficiente para evitar que se produzcan manifestaciones clínicas importantes que ocasionen el AO; no obstante, las mujeres heterocigotas pueden presentar algún grado de mosaicismo en la pigmentación observada en el fondo de ojos y un patrón de autofluorescencia característico $^{17-20}$. La forma de AOC más frecuente, es la asociada a mutación del gen OCA1, en que el compromiso cutáneo es significativo; pero hay otras formas en que la pigmentación de piel puede ser prácticamente normal, como ocurre en la forma asociada al 
Buscar otras causas de movimientos oculares paroxísticos.

Evaluación neurológica.

Eventual estudio neuroimágenes

Lactante con movimientos oculares paroxísticos

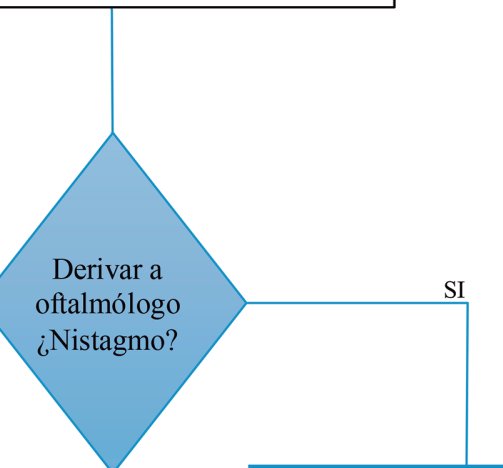

Descartar compromiso neurológico con examen clínica y neuroiomágenes

Si el examen neurológico es normal, se

Antecedentes personales

Examen físico y neurológico completo

Antecedentes familiares

plantea nistagmo idiopático

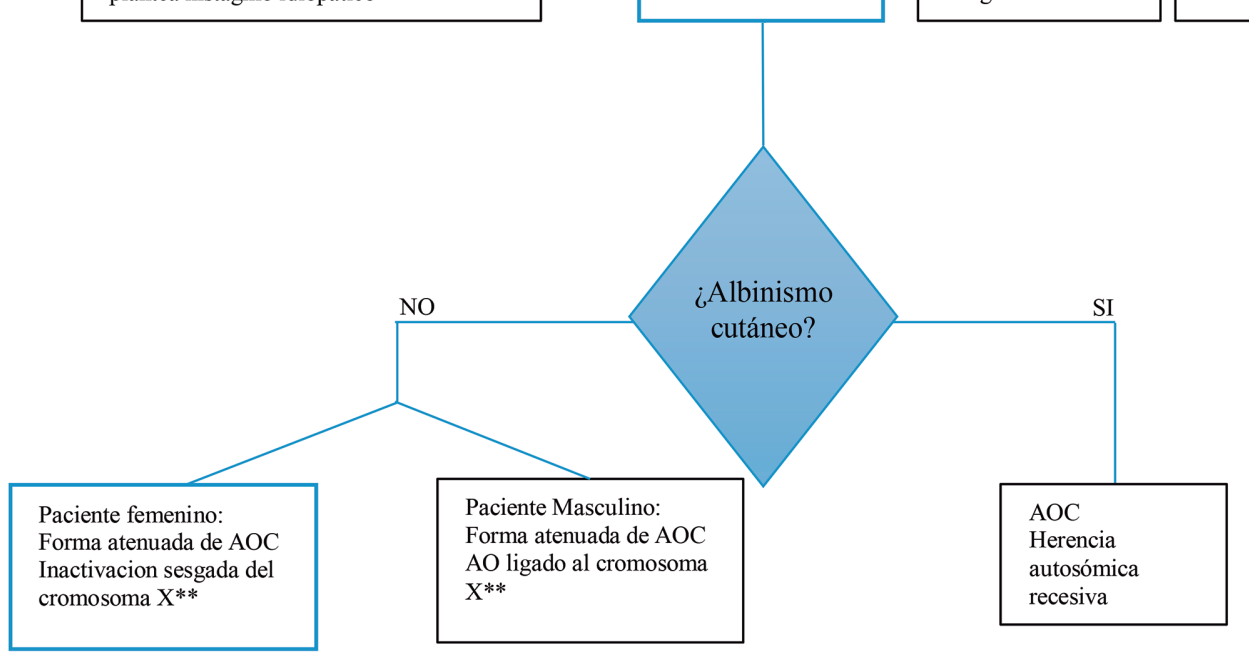

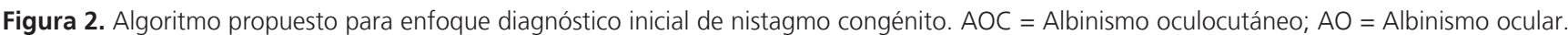
*El oftalmólogo hace el diagnóstico diferencia del transiluminación, considerando otras patologías como uveitis, trauma, disgenesia de segmento anterior u otras. **En contexto sindromático, considerar sindromes de "dilución pigmentaria" como el Sd. de Hermansky- Pudlak, Sd. de ChédiakHigashi y el Sd. de Griscelli. 
gen OCA2, ambas formas son autosómicas recesivas. También forman parte del diagnóstico diferencial del AO el síndrome de Hermansky- Pudlak, síndrome de Chédiak-Higashi, en que el albinismo se asocia a un espectro sindromático ${ }^{13}$.

En nuestro caso, en que se descartó compromiso cutáneo, no había antecedentes familiares de albinismo ni de nistagmo y el examen clínico y la autofluorescencia fueron normales en ambos padres, se planteó como alternativas un AO ligado al $\mathrm{X}$, con manifestaciones fenotípicas que pudieran deberse a una inactivación sesgada del cromosoma X, o alguna forma atenuada de AOC. En la literatura hay escasos casos reportados de $\mathrm{AO}$ en pacientes de sexo femenino ${ }^{21,22}$. El examen genético habria ayudado a dilucidar el diagnóstico diferencial y la futura planificación familiar, lo que no fue considerado como necesario por sus padres, al entender el albinismo como una condición no discapacitante, con una evolución no progresiva. Autores plantean que el de panel genético tiene un rendimiento inferior al $50 \%{ }^{23}$. La costo-efectividad del examen podría variar a futuro gracias al avance en técnicas de secuenciación y la baja progresiva en sus costos.

La intervención temprana multidisciplinaria en pacientes con nistagmo debido a albinismo, permite disminuir las complicaciones y mejorar la calidad de vida. Si bien, a la fecha no se cuenta con un tratamiento curativo $^{11}$, el manejo se centra en corrección de las comorbilidades oftalmológicas, cutáneas, y las alteraciones del desarrollo, secundarias a las dificultades en el contacto visual y a la adopción de posturas cervicales anómalas ${ }^{3,4}$. En nuestro caso, el manejo se hizo con lentes y terapia kinésica, destacando la mejoría en su agudeza visual, postura cefálica y habilidades de coordinación, con un neurodesarrollo normal a la edad de tres años. La evolución favorable de nuestra paciente coincide con reportes de seguimiento de niños con albinismo. Dijkstal y cols. demostraron que la visión binocular mejora durante la etapa escolar ${ }^{24}$, mientras que Kutzbach y cols, reportaron que la mayoría de los portadores tendría un desarrollo neurológico y desempeño académico normal, independiente de las dificultades visuales y la mayor prevalencia de síndrome de déficit atencional con hiperactividad ${ }^{25}$.

\section{Conclusiones}

El nistagmo congénito muchas veces representa un desafío diagnóstico, dado que no siempre es evi- dente al momento del nacimiento, pudiendo manifestarse como movimientos oculares paroxísticos, como ocurrió en el caso clínico presentado. El nistagmo secundario a albinismo, en ausencia de compromiso cutáneo, especialmente en pacientes de sexo femenino, representa un desafío adicional, debido al tipo de herencia predominante en el AO que es ligada al cromosoma X, planteándose como diagnóstico diferencial las formas muy atenuadas de AOC. Si bien el diagnóstico es clínico, el estudio genético permite confirmar la etiología, sin ser, en opinión de los autores, un examen imprescindible ${ }^{23}$. La pesquisa oportuna e intervención multidisciplinaria determinan un mejor pronóstico.

\section{Responsabilidades Éticas}

Protección de personas y animales: Los autores declaran que los procedimientos seguidos se conformaron a las normas éticas del comité de experimentación humana responsable y de acuerdo con la Asociación Médica Mundial y la Declaración de Helsinki.

Confidencialidad de los datos: Los autores declaran que han seguido los protocolos de su centro de trabajo sobre la publicación de datos de pacientes.

Derecho a la privacidad y consentimiento informado: Los autores han obtenido el consentimiento informado de los padres (tutores) de la paciente y/o sujetos referidos en el artículo. Este documento obra en poder del autor de correspondencia

\section{Conflicto de intereses}

Los autores declaran no tener conflicto de intereses.

\section{Agradecimientos}

Los autores agradecen a los padres de la paciente por su colaboración en el seguimiento de la niña y en la elaboración del presente manuscrito. Agradecen además a la Kinesióloga Maria Macarena Donoso F. por compartir su experiencia clínica en la atención de la paciente. 


\section{Referencias}

1. Papageorgiou E, McLean R, Gottlob I, Nystagmus in Childhood. Pediatr Neonatolo 2014; 55: 341-51.

2. Abadi V, Bjerre A, Motor and sensory characteristics of infantile nystagmus. BR J Ophthalmol 2002;86:1152-60.

3. Noval S, González-Manrique M, Rodríguez-Del Valle JM, RodríguezSánchez JM. Abnormal Head Position in Infantile Nystagmus Syndrome. ISRN Ophthalmol. 2011;1-7.

4. Hertle RW, Zhu X. Oculographic and clinical characterization of thirty-seven children with anomalous head postures, nystagmus, and strabismus: the basis of a clinical algorithm. J AAPOS. 2000;4(1):25-32.

5. Sarvananthan N, Surendran M, Roberts EO, et al. The Prevalence of Nystagmus: The Leicestershire Nystagmus Survey. Invest Ophthalmol Vis Sci 2009;50(11):5201-6.

6. Repka MX, Friedman DS, Katz J, Ibironke J, Giordano L, Tielsch JM. The prevalence of ocular structural disorders and nystagmus among preschool-aged children. J AAPOS 2012;16:182-4.

7. Bertsch M, Floyd M, Kehoe T, Pfeifer W, Drack A. The clinical evaluation of infantile nystagmus: What to do first and why. Ophthalmic Genet 2017;38:(1):2233.

8. Lazcano-Gómez G, Fuentes-Cataño C, Villanueva-Mendoza C. Etiología del nistagmo congénito o infantil. Ruta diagnóstica. Rev Mex Oftalmol 2010:84(1):49-54.
9. Kamaraj B, Purohit R. Mutational Analysis of Oculocutaneous Albinism: A Compact Review. BioMed Res Int 2014;905472.

10. Preising MN, Forster H, Gonser M, Lorenz B, Screening of TYR, OCA2, GPR143, and MC1R in patients with congenital nystagmus, macular hypoplasia, and fundus hypopigmentation indicating albinism. Mol Vis 2011;17:93948.

11. Hertle R, Albinism: Particular Attention to the Ocular Motor System. Middle East Afr J Ophthalmol 2013;20(3):248-55.

12. Lewis RA. Ocular Albinism, $\mathrm{X}$-Linked. 2004 [Updated 2015 Nov 19]. In: Adam MP, Ardinger HH, Pagon RA, et al., editors. GeneReviews ${ }^{\circledR}$ [Internet]. Seattle (WA): University of Washington, Seattle; 1993-2019. Available from: https://www. ncbi.nlm.nih.gov/books/NBK1343/ [accedido el 11.11.2019].

13. Grønskov K, Ek J, Brondum-Nielsen K. Oculocutaneous albinism, Orphanet J Rare Dis 2007;2:43

14. Ohlsson J, Villarreal G, Sjöström A, Abrahamsson M, Sjöstrand J. Visual acuity, residual amblyopia and ocular pathology in a screened population of 12-13-year-old children in Sweden. Acta Ophthalmol Scand 2001;79(6):589-595.

15. Rosenberg T, Schwartz M. X-linked ocular albinism: prevalence and mutations-a national study. Eur J Med Genet. 1998;6(6):570.

16. Bassi MT, Schiaffino MV, Renieri A, et al. Cloning of the gene for ocular albinism type 1 from the distal short arm of the $\mathrm{X}$ chromosome. Nat Genet 1995; 10:13-9.

17. Falls HF. Sex linked ocular albinism displaying typical fundus changes in the female heterozygote. Am J Ophthalmol 1951; 34:41-50.

18. Schiaffino MV, Bassi MT, Galli L, et al. Analysis of the OA1 gene reveals mutations in only one third of patients with X-linked ocular albinism. Hum Mol Genet 1995;4:2319-25.

19. Mauri L, Manfredini E, Del Longo A. Clinical Evaluation and molecular screening of a large consecutive series of albino patients. J Hum Genet 2017;62(2):277-90.

20. Rodanant N, Bartsch DU, Bessho K, Freeman WR. Autofluorescence image in ocular albinism. Retina. 2003;23(2):265-6.

21. Scialfa A. Ocular albinism in a female. Am J Ophthalmol 1972;73(6).

22. Pearce WG, Johnson GJ, Gillan JG. Nystagmus in a female carrier of ocular albinism. J Med Genet. 1972;9(1):126-9.

23. O'Gorman L, Norman CS, Michaels $\mathrm{L}$, et al. A small gene sequencing panel realises a high diagnostic rate in patients with congenital nystagmus following basic phenotyping. Scientific reports. 2019;9(1):1-8.

24. Dijkstal JM, San Cooley S, Holleschau AM, King RA, Summers CG. Change in visual acuity in albinism in the early school years. Journal of pediatric ophthalmology and strabismus. 2012;49(2):81-6.

25. Kutzbach BR, Summers CG, Holleschau AM, MacDonald JT. Neurodevelopment in children with albinism. Ophthalmology. 2008;115(10):1805-8. 